\title{
Microwave assisted preparation of bismuth oxyhalide microflowers comprised of nanolayers and investigation of its photocatalytic activity
}

\author{
Azadeh Tadjarodia $^{\mathrm{a}, *}$, Mohammad Moghaddasi ${ }^{\mathrm{a}}$, Keyvan Bijanzad ${ }^{\mathrm{a}}$, Omid Akhavan $^{\mathrm{b}, \mathrm{c}}$ \\ ${ }^{a}$ Research Laboratory of Inorganic Materials Synthesis, Department of Chemistry, Iran \\ University of Science and Technology, Narmak, Tehran 16846-13114, Iran \\ (E-mail:tajarodi@iust.ac.ir) \\ ${ }^{b}$ Department of Physics, Sharif University of Technology, P.O. Box 11155-9161, Tehran,Iran, \\ ${ }^{c}$ Institute for Nanoscience and Nanotechnology, Sharif University of Technology, P.O. Box \\ 14588-89694, Tehran, Iran
}

\begin{abstract}
Today, bismuth based oxides, which have already been used as pigments and catalysts, are showing excellent photocatalytic performances. In this field, ternary bismuth oxyhalides (BiOX) are flourishing promptly. Bismuth nitrate pentahydrate and sodium chloride were utilized to fabricate the product in a domestic microwave instrument. The SEM images showed the microflowers which were comprised of nanolayers with the thickness of about 29 nm. From energy dispersive X-ray analysis, its empirical formula was estimated to be $\mathrm{Bi}_{2.27} \mathrm{O}_{9.31} \mathrm{Cl}$. Fourier transform infrared spectroscopy showed that the peak at $567 \mathrm{~cm}^{-1}$ was attributed to the stretching vibration of Bi-O. Diffuse reflectance spectrum showed band gap energy of $3.5 \mathrm{eV}$ for this compound. It showed a high photocatalytic performance in photodegradation of Rhodamine B.
\end{abstract}

Keywords: Microwave sytnthesis, Bismuth oxychloride, photocatalyst, Rhodamine B.

\section{Introduction}

In the recent years, Kudo et al. discovered that bismuth-based oxide semiconductors could show high photocatalytic activity because the $\mathrm{Bi} 6 \mathrm{~s}$ and $\mathrm{O} 2 \mathrm{p}$ levels form largely dispersed hybridized valence bands [1]. The band gap of $\mathrm{BiOX}$ is strictly dependent on the halide participated in its composition and ranges from 3.19-3.44, 2.64-2.91 and 1.77-1.92 eV, respectively for $\mathrm{Cl}, \mathrm{Br}$ and $\mathrm{I}$ [2]. In this field, so far, researchers have also investigated photocatalytic activities of various bismuth compounds such as: $\mathrm{Ag} / \mathrm{AgI} / \mathrm{BiOI}$ [3], $\mathrm{Bi}_{2} \mathrm{~S}_{3}$ [4], $\mathrm{Bi}_{2} \mathrm{Ti}_{2} \mathrm{O}_{7}$ [5], N-doped ( $\left.\mathrm{BiO}\right)_{2} \mathrm{CO}_{3}$ [6], $\mathrm{Bi}_{5} \mathrm{O}_{7} \mathrm{NO}_{3}$ [7], $\mathrm{BiOI}$ [8], $\mathrm{Bi}_{2} \mathrm{O}_{3}, \mathrm{BiVO}_{4}, \mathrm{Bi}_{2} \mathrm{WO}_{6}[9,10$ ], $\mathrm{BiNbO}_{4}$ and $\mathrm{BiTaO}_{4}$ [11] and Black BiOCl [12]. Among different ternary bismuth based 
photocatalysts, bismuth oxyhalides ( $\mathrm{BiOX}$ ) have captivated the interest of researchers enormously. $\mathrm{BiOCl}$ was the first oxyhalide to be used as photocatalyst. It is a narrow band gap $(2.87 \mathrm{eV})$ semiconductor which can be used as a catalyst (e.g. oxidative coupling of methane) and as a pigment in the cosmetic industry [13].

\section{Experimental section}

Bismuth nitrate pentahydrate (98.0\%) was purchased from SAMCHUN CHEMICALS, Korea. All the other reagents were purchased from Merck Company and used without further purification. Rhodamine B (RhB) was used as the model pollutant. Deionized water was used to prepare all the solutions. Bismuth nitrate pentahydrate and sodium chloride were utilized to fabricate the product in a domestic microwave instrument (Samsung GE 280) at 180 Watts for 23 minutes.

\section{Results and discussion}

Fourier transform infrared spectroscopy showed that the peak at $567 \mathrm{~cm}^{-1}$ was attributed to the stretching vibration of Bi-O. Other peaks were related to carboxyl and/or hydroxyl surface functional groups.

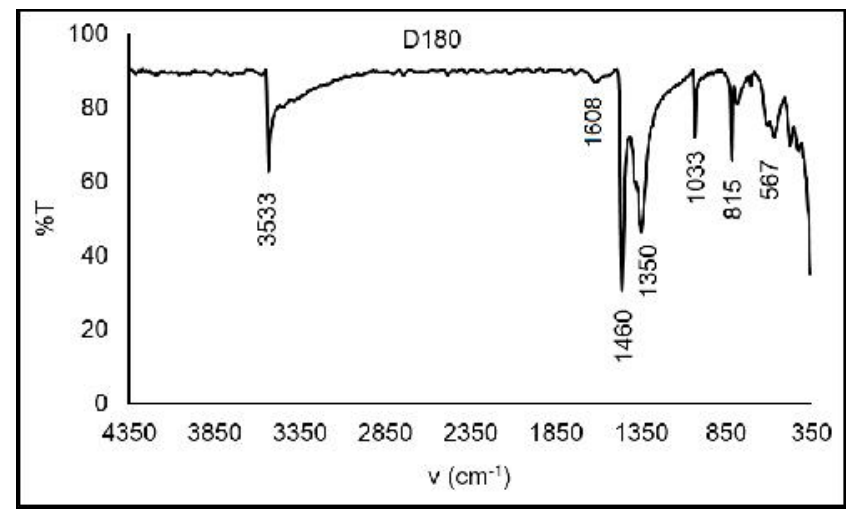

Fig. 1. FT-IR spectrum of the bismuth oxychloride nanostructure.

As it can be seen in Fig. 2, the reflections of the product could be indexed to the tetragonal phase of $\mathrm{BiOCl}$ pattern with the space group of $\mathrm{P} 4 / \mathrm{nmm}$ and lattice parameters of $\mathrm{a}=3.89$, $b=3.89$ and $c=7.37 \AA, \alpha=\beta=\gamma=90^{\circ}$ (JCPDS card no. 00-006-0249). 


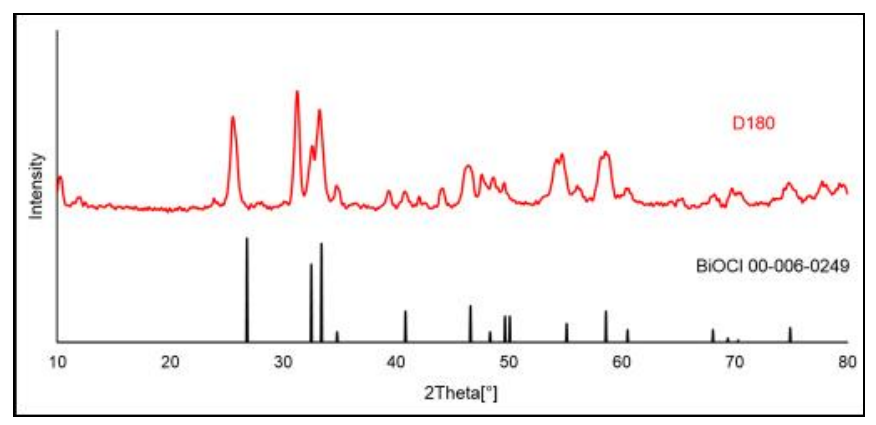

Fig. 2. XRD patterns of bismuth oxychloride nanostructure and BiOCl (JCPDS file no. 00-006-0249).

The SEM images showed the microflowers were comprised of nanolayers with the thickness of about $29 \mathrm{~nm}$. They revealed a rose-like morphology in which the nanosheets were constructing components.
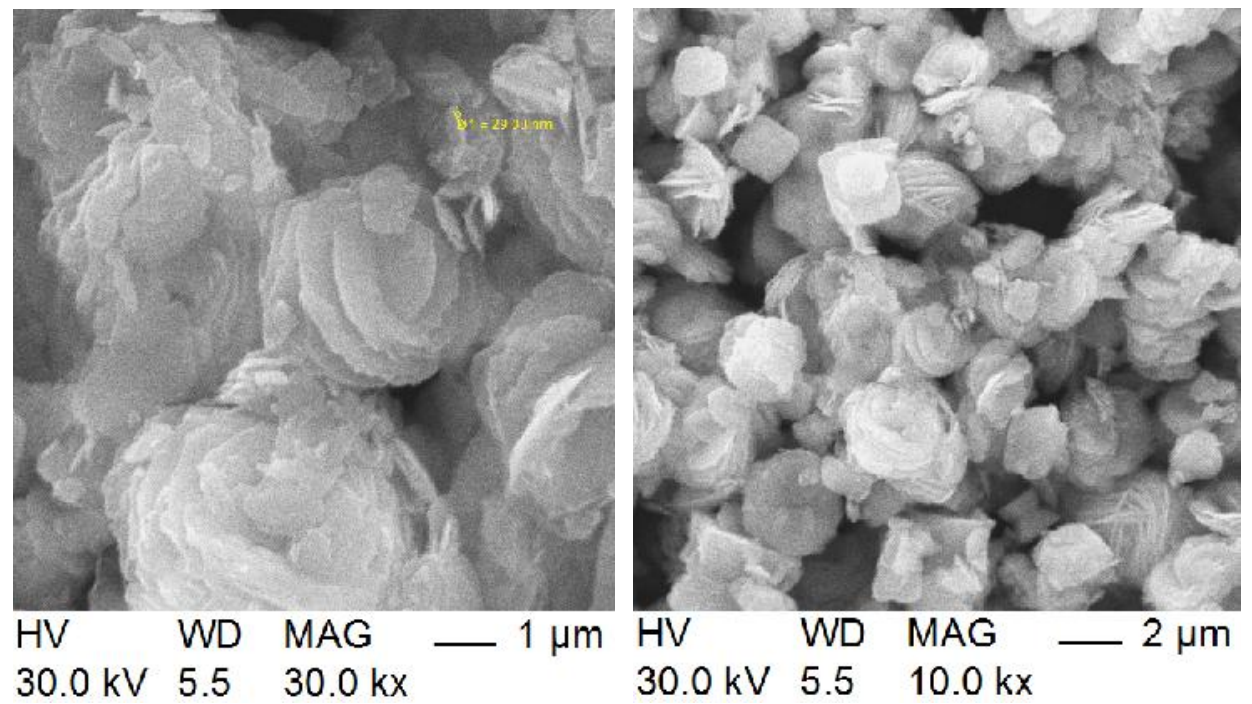

Fig. 3. SEM images of bismuth oxychloride nanostructure

From energy dispersive X-ray analysis, its empirical formula was estimated to be $\mathrm{Bi}_{2.27} \mathrm{O}_{9.31} \mathrm{Cl}$. 


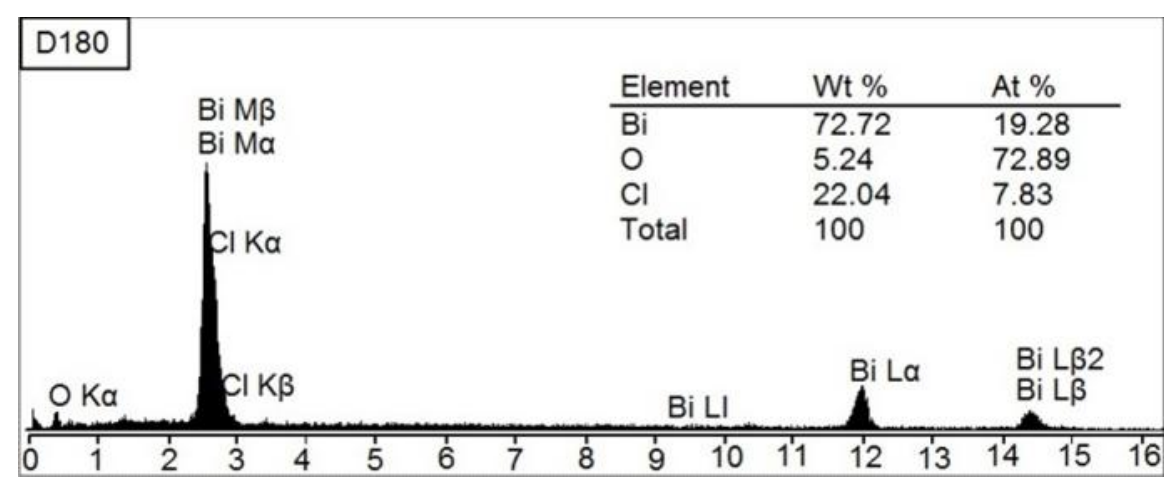

Fig. 4. EDX analysis of bismuth oxychloride nanostructure.

With a band gap energy of $3.53 \mathrm{eV}$, it showed a high photocatalytic performance in photodegradation of rhodamine $\mathrm{B}(\mathrm{RhB})$ and more than $70 \%$ of the dye molecules were degraded in $5 \mathrm{~h}$. According to previous studies, a possible mechanism for the degradation of $\mathrm{RhB}$ is based on deethylation and carboxylation of the organic dye molecules and the intermediates where the dye is finally mineralized to $\mathrm{CO}_{2}$ and $\mathrm{H}_{2} \mathrm{O}$. The gradual blue-shift of the main absorption peak of RhB was ascribed to the step-by-step deethylation of the dye molecules, and the reduction in absorbance intensity revealed the destruction of the conjugated structure [14].

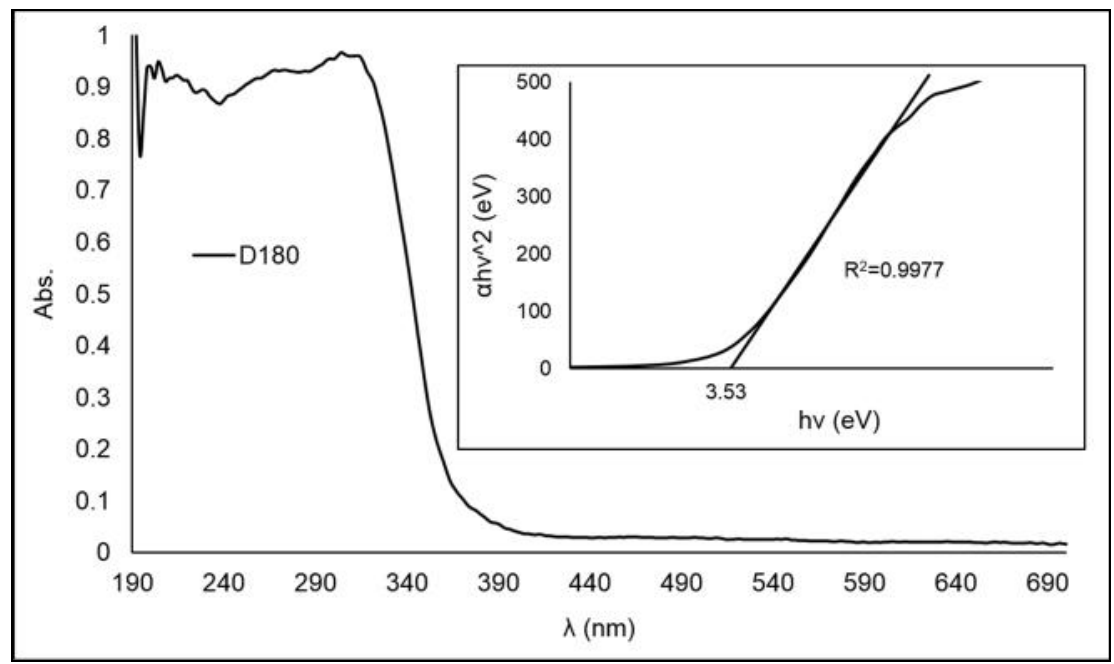

Fig. 5. UV-Vis diffuse reflectance spectrum of bismuth oxychloride (the inset shows plot of $(\alpha \mathrm{h} v)^{2}$ vs. $\mathrm{h} v$ for corresponding product). 


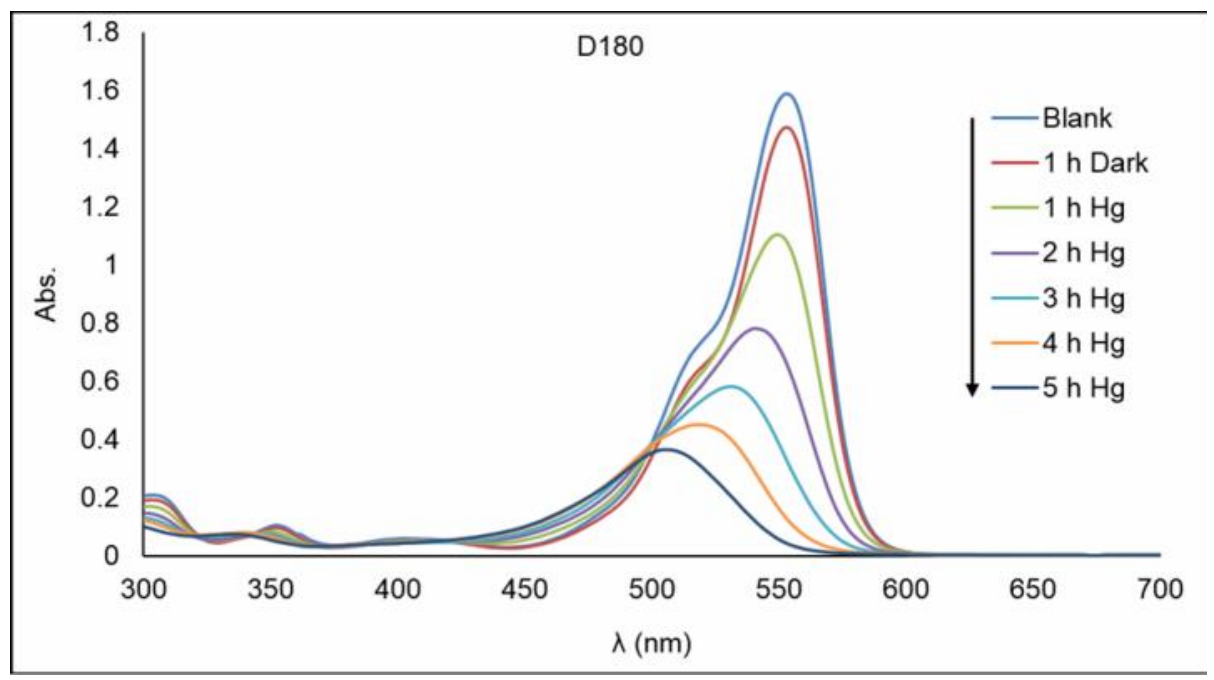

Fig. 6. UV absorption spectra of $\mathrm{RhB}$ in presence of $0.05 \mathrm{~g}$ of photocatalyst under Mercury lamp for $5 h$.

\section{Conclusion:}

Bismuth nitrate pentahydrate and sodium chloride were utilized to prepare microflowers of $\mathrm{Bi}_{2.27} \mathrm{O}_{9.31} \mathrm{Cl}$ in a domestic microwave instrument. The SEM images showed the microflowers were comprised of nanolayers. With a band gap energy of $3.5 \mathrm{eV}$, it showed a high photocatalytic performance in photodegradation of Rhodamine B. This research shows that the microwave method is a green and facile method to synthesize new classes of semiconductors.

\section{References:}

[1] S. Sun, W. Wang, L. Zhang, L. Zhou, W. Yin, M. Shang, Environ. Sci. Technol. 43 (2009) 2005.

[2] B. Zhang, G. Ji, Y. Liu, M. A. Gondal, X. Chang, Catal. Commun., 36 (2013) 36, 25.

[3] J. Cao, Y. Zhao, H. Lin, B. Xu, S. Chen, J. Solid State Chem. 206 (2013) 38.

[4] B. Pejova, A. Tanuševski, I. Grozdanov, J. Solid State Chem. 178 (2005) 1786.

[5] J. Hou, S. Jiao, H. Zhu, R.V. Kumar, J. Solid State Chem. 184 (2011) 154.

[6] F. Dong, Y. Sun, M. Fu, W.-K. Ho, S.C. Lee, Z. Wu, Langmuir. 28 (2011) 766.

[7] S. Yu, G. Zhang, Y. Gao, B. Huang, J. Colloid Interface Sci. 354 (2011) 322.

[8] Y. Wang, K. Deng, L. Zhang, J. Phys. Chem. C. 115 (2011) 14300.

[9] T. Saison, N. Chemin, C. Chanéac, O. Durupthy, V.r. Ruaux, L. Mariey, F.o. Maugé, P. Beaunier, J.-P. Jolivet, J. Phys. Chem. C. 115 (2011) 5657.

[10] S. Dong, C. Yu, Y. Li, Y. Li, J. Sun, X. Geng, J. Solid State Chem. 211 (2014) 176.

[11] H.-F. Zhai, A.-D. Li, J.-Z. Kong, X.-F. Li, J. Zhao, B.-L. Guo, J. Yin, Z.-S. Li, D. Wu, J. Solid State Chem. 202 (2013) 6.

[12] Y. Li, C. Li, Z. Zhang, Y. Zhang, X. Sun, H. Si, J. Zhang, Solid State Sci. 34 (2014) 107. 
[13] S. Shenawi-Khalil, V. Uvarov, S. Fronton, I. Popov, Y. Sasson, Appl. Catal. B. 117-118 (2012) 148.

[14] W. Cui, W. An, L. Liu, J. Hu, Y. Liang, J. Solid State Chem. 215 (2014) 94. 